\title{
Sex Hormone-binding Globulin (SHBG) and Estradiol Cross-talk in Breast Cancer Cells
}

\author{
N. Fortunati ${ }^{1}$ \\ M. G. Catalano ${ }^{2}$
}

\section{Abstract}

Sex Hormone-binding Globulin (SHBG) is a plasma glycoprotein that regulates the action of steroid hormones at several levels. SHBG regulates the availability of free androgens and estradiol to hormone-responsive tissues. Moreover, SHBG is also part of a novel steroid signaling system. We report here on the mechanism of action and the biological effects of SHBG in breast cancer cells, especially distinguishing cross-talk between membraneinitiated SHBG and estradiol pathways. After interacting with a specific binding site on breast cancer cell membranes, SHBG ac- tivates a specific pathway, and by cAMP induction, inhibits estradiol-mediated activation of ERK. Both estradiol and SHBG membrane-initiated pathways involve cross-talk at MAP kinase level with the ultimate result of inhibiting estradiol-mediated cell growth and antiapoptosis. On the basis of reported evidence, we suggest that SHBG is one of the regulators of growth and apoptosis of estrogen-dependent breast cancer cells.

\section{Key words}

SHBG · breast cancer · estradiol effect · SHBG membrane binding site

\section{Introduction}

Breast cancer development and progression are strongly influenced by estradiol, which acts by inducing cell proliferation and inhibiting apoptosis. Agents able to modify estradiol action in target cells as well as their circulating levels and metabolic pathways are of great interest for therapeutic intervention. Among them, sex hormone-binding globulin (SHBG) has received increasing attention over time due to its unique ability to bind circulating estradiol at high affinity [1] and regulate estradiol action within the cell [2].

Levels of circulating SHBG have been widely studied in breast cancer patients together with other potential risk factors. In postmenopausal breast cancer patients, SHBG plasma levels are reduced or at least at the lower end of normal range [3-5]; in these patients, the free fraction of estradiol is, on the other hand, increased in relation to an absolute or relative reduction of SHBG [6]. Moreover, a recent reanalysis of nine prospective studies on breast cancer risk concluded that SHBG in postmenopausal women is associated with a decrease in breast cancer risk [7].

It should also be remembered that in the late 70s some authors reported that SHBG plasma levels were increased in estrogen receptor-positive breast cancer patients [8-10].

In addition, some authors reported the presence of immunoreactive SHBG in breast cancer cells and tissue sections [11-15], as well as the demonstration of SHBG mRNA [16]. Unfortunately, studies showing the presence of SHBG mRNA failed to provide conclusive evidence for the presence of the protein, and conclusions based on experiments using human tissue section are ambiguous overall. Lastly, no convincing functional role for this hypothetical intracellular protein in breast cancer has ever been delineate.

Affiliation

${ }^{1}$ Oncological Endocrinology, ASO San Giovanni Battista, Turin, Italy

${ }^{2}$ Department of Clinical Physiopathology, University of Turin, Turin, Italy

Correspondence

Nicoletta Fortunati, M.D. · Lab. Endocrinologia Oncologica · Via Genova $3 \cdot 10126$ Torino · Italy

Fax: +39-011-670.5366 · E-Mail: nfortunati@molinette.piemonte.it

Received 1 August $2005 \cdot$ Accepted after revision 21 November 2005

Bibliography

Horm Metab Res 2006; 38: 236-240 @ Georg Thieme Verlag KG Stuttgart · New York .

DOI 10.1055/s-2006-925337 · ISSN 0018-5043 
Taking all these data into account, it is clear that some functional relationship between SHBG and breast cancer must exist. Provided that the regulation of the bioavailable free estradiol fraction has a meaningful role, at least in postmenopausal women, the direct effect of SHBG on estradiol action in breast cancer cells and the pathways triggered will be the subject of the present discussion.

\section{SHBG Binds to Breast Cancer Cell Membrane}

The suggestion that SHBG could play an active role at the level of the cell membrane is driven by reports regarding the presence of specific and high-affinity binding sites for SHBG on cell membranes from endometrium [17-19], prostate [20], human placenta [21], and epididymis [22]. More recently, binding sites for SHBG have also been described in medial preoptic area and medial basal hypothalamus in rats [23], while SHBG-receptor interaction has been suggested as responsible for many steroid effects in brain and peripheral cells [24,25]. Furthermore, specific binding sites for SHBG were also described on membranes of estrogendependent MCF-7 breast cancer cells [26,27].

The binding characteristics of SHBG to cell membranes are consistent with the presence of a receptor structure. In fact, all the authors described binding that is highly specific and dependent on time and temperature in the different tissues. Unfortunately, no data are available on receptor structure, and its gene has not been identified and characterized yet. Nonetheless, our knowledge about the binding modalities of SHBG to membranes is quite exhaustive. As elegantly described by Hryb and coworkers $[28,29]$, we are aware that only steroid-free SHBG binds to cell membranes; on the other hand, where sex steroids bind to SHBG first, they prevent SHBG-cell interaction; the magnitude of the inhibition is directly proportional to the magnitude of the association constant for the steroid-SHBG interaction. Once bound to the membrane site, SHBG binds steroids with equal affinity to that in solution. Lastly, this mechanism of successive binding is crucial in triggering the biological effects of SHBG in target cells as will be discussed below.

The SHBG domain that interacts with cell membranes has also been identified, at least in part [30]. It is localized to a ten-amino-acid stretch (TWDPEGVIFY) in the amino-terminal end of the SHBG molecule. This region, also referred to as laminin-G domain of human SHBG [30,31], is the most highly conserved portion of the molecule across species [30] and in related proteins such as protein S, laminin A, merosin, and Drosophila crumbs protein $[30,32,33]$.

Moreover, the biological effect of SHBG on MCF-7 breast cancer cell proliferation (which will be described in detail in the next paragraphs) depends on the structural integrity of the 0 -glycosylation site in $\mathrm{Thr}^{7}$ [34]. While SHBG mutants lacking the two $\mathrm{N}$ glycosylation sites located in the carboxy-terminus of the protein show activity no different from breast cancer cell proliferation seen in wild type protein, the mutant SHBG lacking $\mathrm{Thr}^{7} \mathrm{O}$-glycosylation in its amino terminus fails to block cell growth.
Taking all these observations together, SHBG clearly interacts with cell membranes at its amino-terminus portion, the laminin-G domain. The carbohydrate chain in this domain contributes to its structural stability, and allows correct binding to cell membranes and induction of SHBG's biological effects in breast cancer cells.

\section{SHBG and Estradiol Pathways Cross-talk after Membrane Initiation}

As mentioned above, SHBG recognizes a specific binding site located on cell membranes of estrogen-dependent MCF-7 breast cancer cells $[26,27]$. On the other hand, no binding was detectable on MDA-MB 231 breast cancer cells negative for estrogen receptor alpha $(E R \alpha)$ and generally considered estrogen-insensitive [35]. Furthermore, when SHBG binding was evaluated on tissue samples from breast cancer patients, it was detectable on $75 \%$ of ER $\alpha$-positive samples but only in $37 \%$ of ER $\alpha$-negative samples. In addition, samples binding SHBG and expressing ER $\alpha$ were also characterized by a significantly lower proliferation rate than samples not able to bind SHBG. So far, it appears that SHBG action in breast cancer cells must be strictly linked to estradiol effects in these cells [36].

A membrane-initiated effect of estradiol has been hypothesized for a long time, but was not described in detail until recently. In fact, beside the classical pathway mediated by the nuclear ER, estradiol binds to a putative membrane ER [37] and triggers specific intracellular signal transduction pathways [38]. There is membrane-initiated pathway cross-talks at several points with pathways initiated by growth factors such as EGF [39]. Both pathways - one induced by growth factors and mediated by membrane ER - induce activation of MAP kinase that transmits and amplifies signals involved in both cell proliferation and apoptosis [4042]. Moreover, MAP kinase can directly catalyze the phosphorylation of serine 118 of nuclear ER $\alpha$, thus increasing ER transcriptional efficiency [43]. The result of this multiple-site cross-talk is strong signal amplification and, finally, potent stimulation of cell growth and inhibition of apoptosis.

After binding to MCF-7 cell membranes, SHBG triggers downstream signaling, which starts with an increase in intracellular cAMP. The accumulation of cAMP induced by SHBG has been described both in prostate $[44,45]$ and breast $[35,46]$ cancer cells; the main difference between the two cell types was reported to lie in the steroid that activated SHBG after membrane binding; DHT in the prostate and estradiol in breast cancer cells. SHBG induced an elevation in intracellular cAMP, suggesting that the putative SHBG receptor is coupled to a G-protein [47], but evidence for it is still lacking. However, the biological effects of SHBG appeared to be mediated by cAMP and its target PKA. In fact, biological effects of SHBG $[48,49]$ are lost in MCF-7 cells when PKA is blocked by the specific inhibitor, PKI (6-22).

The immediate target of increased PKA in breast cancer cells after SHBG activation has not yet been identified, but new data suggest that increased PKA levels can inhibit the MAP kinase pathway. In fact, we demonstrated that SHBG, after membrane binding, counteracts the estradiol-induced ERK activation [50], 


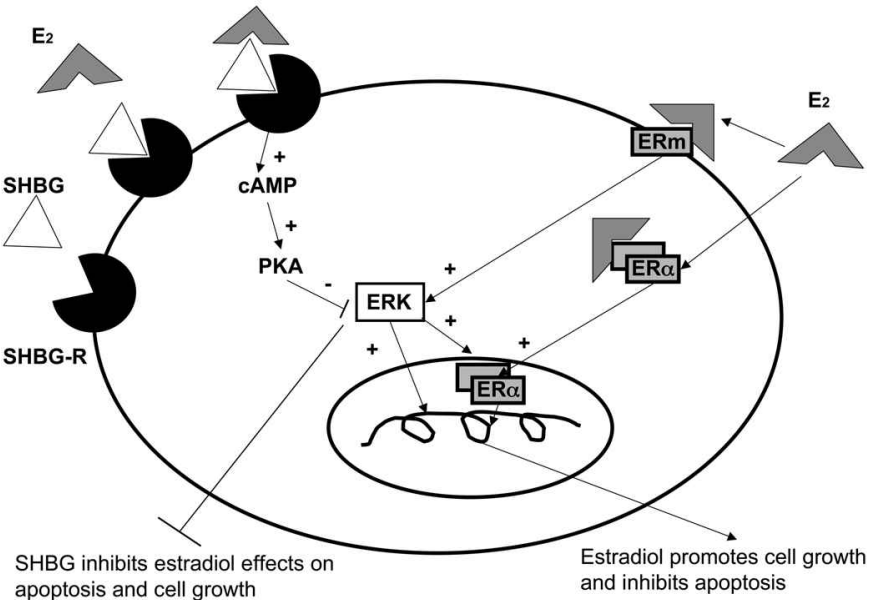

Fig. 1 SHBG and estradiol pathways in breast cancer cells. In its steroid-free configuration, SHBG binds to its receptor (SHBG-R). After binding to estradiol $\left(E_{2}\right)$, the complex activates adenylyl cyclase, leading to cAMP generation, which in turn inhibits ERK. Estradiol can also bind either to its membrane receptor (ERm), leading to ERK activation, or to the intracellular $E R(E R \alpha)$, activating the genomic pathway. Therefore, SHBG and estradiol membrane-initiated pathways have opposite effect on cell growth and apoptosis.

which is one of the MAP kinases involved in cell proliferation control. ERK activity is suppressed by both CAMP and PKA as reported by Filardo and co-workers [51]. Lastly, inhibition of ERK may result in both removal of anti-apoptotic effects caused by estradiol as we have demonstrated [50], and reduction of ER $\alpha$ transcription efficiency [43]. The evoked pathways and the postulated cross-talk between estradiol and SHBG in breast cancer cells are reported in Fig. 1.

\section{SHBG Modulates Estradiol-regulated Effects in Breast Cancer Cells}

Membrane-initiated SHBG and estradiol pathways are therefore likely to be subject to cross-talk in breast cancer cells. As mentioned above, SHBG is able to counteract estradiol induction of MAP kinase, thus reducing estradiol efficacy. The SHBG-estradiol cross-talk is therefore likely to interfere with biological estradiol effects in breast cancer cells.

One of the markers of estradiol efficacy in breast cancer is the induction of the progesterone receptor (PR) expression. In MCF-7 cells treated with estradiol, the steroid greatly upregulates PR levels measured either by gene expression or by functioning protein [52,53]. When MCF-7 cells are treated with SHBG first and then with estradiol, both RNA and protein PR levels do not change with respect to basal levels; the induction of PR by estradiol is thus inhibited by SHBG [48]. Moreover, the effect of SHBG on estradiol regulation of $P R$ is highly specific for the protein since it is abolished by anti-SHBG antibody and depends on the affinity of estradiol for SHBG (DES, estrogenic compound binding to the estrogen receptor but not to SHBG, is able to up-regulate PR even in the presence of SHBG).

Beside apart from the anti-estrogenic effect on PR, SHBG has been widely demonstrated to inhibit estrogen-dependent cell growth. Estradiol is well known to induce and maintain the proliferation of breast cancer cells [54], and the effect of estradiol on breast cancer cell growth is due to the activation of multiple pathways. First the hormone, through its nuclear receptor ER $\alpha$, regulates the transcription and expression of many genes involved in cell proliferation, inducing positive regulators of cell growth while inhibiting negative regulators $[55,56]$. Second, estradiol by interacting with its membrane receptor (ERm) [37] activates ERK and inhibits Jun kinase, causing the induction of bcl2 and thus the inhibition of apoptosis in MCF-7 breast cancer cells [38]. Since one of the first descriptions of the SHBG binding site on MCF-7 cells [27], interaction between SHBG with its receptor in breast cancer cells was demonstrated to inhibit estradiol-induced cell proliferation. Some years later, we confirmed our previous observation and described in detail this effect of SHBG in MCF-7 cells [49]. The antiproliferative effect of SHBG is detectable when the correct sequence of binding is followed; that is, first, SHBG binds to its membrane-associated receptor on MCF-7 cells, then estradiol binds at the steroid-binding site of SHBG. Recombinant SHBG produced by MCF-7 cells transfected with human shbg gene was shown to retain all the anti-proliferative properties of the SHBG [34] while, much more interestingly, MCF-7 cells producing SHBG after transfection completely lose their capacity to bind SHBG at membranes.

Furthermore, SHBG was recently reported to inhibit another estradiol effect that has great importance in the control of cell proliferation. As mentioned above, estradiol inhibits apoptosis in MCF-7 cells and does it by interacting with its membrane receptor, ERm [38]. SHBG [50] is capable of reversing the anti-apoptotic effect of estradiol; in MCF-7 cells, it does so when used in physiological concentrations and, again, only when the correct sequence of binding is followed. Moreover, the structural integrity of the 0 -glycosylation site in $\mathrm{Thr}^{7}$ of the amino-terminal end of SHBG is required to block the estradiol antiapoptotic effect [50] as already reported for the inhibition of cell proliferation induction [31]. In addition, SHBG blocks the estradiol anti-apoptotic effect without affecting estradiol-regulated transcription, as demonstrated in a general transcriptional assay using an EREtk-luciferase system [50].

As seen in Fig. 1, we postulate the existence of cross-talk between the SHBG membrane-initiated pathway and estradiol membrane-elicited effects in breast cancer cells. First, SHBG binds to its membrane associated receptor (SHBG-R), and then the glycoprotein itself binds to estradiol activating a second messenger pathway that demonstrates increased cAMP and PKA accumulation. Estradiol alone, on the other hand, after interacting with its own membrane-associated receptor (ERm), stimulates another pathway resulting in ERK phosphorylation, which is finally involved in apoptosis inhibition and cell proliferation induction. As indicated, the former SHBG-stimulated pathway inhibits this ERm-stimulated pathway; the result is that SHBG stimulation inhibits ERK phosphorylation. Therefore, there is an antagonistic relationship between the SHBG and estradiol stimulated effects in breast cancer cells.

Finally, the inhibition of estradiol effects in breast cancer cells by SHBG requires structural characteristics and biochemical events supporting at least two assumptions: first, SHBG might interact 
with cell membranes at a specific site and, second, the SHBG effect is not due to a simple sequestration of circulating estradiol, but requires the activation of specific pathways.

\section{Conclusions}

SHBG is now believed to be a multifunctional protein, and its role in estrogen signaling is of biological relevance. In estrogen-dependent breast cancer, in particular, SHBG functions as a negative modulator of estradiol action and should no longer be considered as a passive player, as its action in breast cancer cells requires an active role. The inhibition of estradiol effects by SHBG requires the interaction of SHBG with cell membranes at a specific site and the activation of specific pathways since this is not due to a simple sequestration of circulating estradiol. Furthermore, both estradiol and SHBG membrane-initiated pathways are subject to cross-talk at MAP kinase level with the ultimate result of inhibiting estradiol-mediated cell growth and antiapoptosis.

The recent evidence supporting the cross-talk between estradiol and SHBG pathways has far-reaching implications for the intracellular signaling network involved in breast cancer initiation and progression. In conclusion, it appears from the growing body of evidence that SHBG is one of the regulators of growth and apoptosis of estrogen-dependent breast cancer cells, with a final antiproliferative effect.

\section{Acknowledgements}

Thanks to: Roberto Frairia, Laura Berta, Marzia Becchis, Alessandra Comba, Elisabetta Benedusi-Pagliano, Federica Fissore, Annamaria Fazzari, Patrizia Ferrera, Mariangela Raineri, Lucia Costantino, Maria Teresa Pugliese; special thanks to Giuseppe Boccuzzi for critical revision of manuscript and valuable suggestions.

\section{References}

${ }^{1}$ Hammond GL. Potential functions of plasma steroid-binding proteins. Trend Endocrinol Metab 1995; 6: 298-340

${ }^{2}$ Fortunati N. Sex hormone-binding globulin: not only a transport protein. What news is around the corner? J Endocrinol Invest 1999; 22: $223-234$

${ }^{3}$ Moore JW, Clark GM, Bulbrook RD, Hayword JL, Murai JT, Hammond GL, Siiteri PK. Serum concentrations of total and non-protein bound estardiol in patients withb breast cancer and in normal controls. Int J Cancer 1982; 29: 17-21

${ }^{4}$ Toniolo PG, Levitz M, Zeleniuch-Jaquotte A, Benerjee S, Koenig KL, Shore RE, Strax P, Pasternack BS. A prospective study of endogenous estrogens and breast cancer in postmenopausal women. J Natl Cancer Inst 1995; 87: 190-197

${ }^{5}$ Zeleniuch-Jacquotte A, Shore RE, Koenig KL, Akhmedkhanov A, Afanasyeva Y, Kato I, Kim MY, Rinaldi S, Kaaks R, Toniolo P. Postmenopausal levels of oestrogen, androgen, and SHBG and breast cancer: long-term results of a prospective study. Br J Cancer 2004; 90: 153-159

${ }^{6}$ Lipworth L, Adami HO, Trichopoulos D, Carlstrom K, Mantzoros C. Serum steroid hormone levels, sex hormone-binding globulin, and body mass index in the etiology of postmenopausal breast cancer. Epidemiology 1996; 7: $96-100$

${ }^{7}$ Key T, Applebay P, Barnes I, Reeves G Endogenous Hormones and Breast Cancer Collaborative Group. Endogenous sex hormones and breast cancer in postmenopausal women: reanalysis of nine prospective studies. J Natl Cancer Inst 2002; 94: 606-616
8 Murayama Y, Sakuma T, Udagawa H, Utsunomiya J, Okamoto R, Asano K. Sex hormone-binding globulin and estrogen receptor in breast cancer: technique and preliminary clinical results. J Clin Endocrinol Metab 1978; 46: $998-1006$

${ }^{9}$ Murayama Y, Utsunomiya J, Takahashi I, Kitamura M, Tominaga T. Sex hormone binding globulin as a reliable indicator of hormone dependence in human breast cancer. Ann Surg 1979; 190: 133-138

10 Plymate SR, Stutz FH, Fariss BL. Relationship betweeen sex hormonebinding globulin and estrogen receptors in breast cancer. J Clin Oncol 1984; 2: $652-654$

${ }^{11}$ Bordin S, Petra PH. Immunocytochemical localization of the sex steroid-binding protein of plasma in tissues of the adult monkey Macaca Nemestrina. Proc Natl Acad Sci USA 1980; 70: 5678-5682

12 Tardivel-Lacombe J, Egloff M, Mazabraud A, Degrelle H. Immunohistochemical detection of the sex steroid-binding plasma protein in human mammari carcinoma cells. Biochem Biophys Res Commun 1984; 118: $488-494$

${ }^{13}$ Sinnecker G, Hiort O, Mitze M, Donn F, Neumann S. Immunohistochemical localization of sex hormone-binding globulin in normal and neoplastic breast tissue. Horm Metab Res 1990; 22: 47-50

${ }^{14}$ Meyer S, Brumm C, Stegner HE, Sinnecker GH. Intracellular sex hormone binding globulin (SHBG) in normal and neoplastic breast tissue - an additional marker for hormone dependency? Exp Clin Endocrinol 1994; 102: 334- 340

${ }^{15}$ Germain P, Egloff M, Kiefer H, Metezeau P, Habrioux G. Use of confocal microscopy to localize the SHBG interaction with human breast cancer cell lines - a comparison with serum albumin interaction. Cell Molec Biol 1997; 43: $501-508$

${ }^{16}$ Moore KH, Bertram KA, Gomez RR, Styner MJ, Matej LA. Sex hormone binding globulin mRNA in human breast cancer: detection in cell lines and tumor samples. J Steroid Biochem Molec Biol 1996; 59: 297-304

${ }^{17}$ Strel'chyonok OA, Avvakumov GV, Survilo LI. A recognition system for sex-hormone-binding protein-estradiol complex in human decidual endometrium plasma membranes. Biochim Biophys Acta 1984; 802: $459-466$

${ }^{18}$ Fortunati N, Fissore F, Fazzari A, Berta L, Giudici M, Frairia R. Sex steroid-binding protein interacts with a specific receptor on human premenopausal endometrium membrane: modulating effect of estradiol. Steroids 1991; 56: $341-346$

${ }^{19}$ Fortunati N, Frairia R, Fissore F, Berta L, Fazzari A, Gaidano G. The receptor for human sex steroid binding protein (SBP) is expressed on membranes of neoplastic endometrium. J Steroid Biochem Mol Biol 1992; 42: 185- 191

${ }^{20}$ Hryb DJ, Khan MS, Rosner W. Testosterone-estradiol-binding globulin binds to human prostatic cell membranes. Biochem Biophys Res Commun 1985; 128 : $432-440$

${ }^{21}$ Avvakumov GV, Survilo LI, Strel'chenok OA. Interaction of blood sex steroid-binding globulin with cell membranes of human decidual tissue. Biokhimiia. 1985; 50: 1155-1161

${ }^{22}$ Gueant JL, Fremont S, Felden F, Nicolas JP, Gerard A, Leheup B, Gerard $\mathrm{H}$, Grignon $\mathrm{G}$. Evidence that androgen-binding protein endocytosis in vitro is receptor mediated in principal cells of the rat epididymis. J Mol Endocrinol 1991; 7: 113-122

${ }^{23}$ Caldwell JD. Evidence of sex hormone binding globulin binding sites in the medial preoptic area and hypothalamus. Horm Metab Res 2001; 33: 7-9

${ }^{24}$ Caldwell JD. A sexual arousability model involving steroid effects at the plasma membranes. Neurosci Biobehav Rev 2002; 26: 13-30

${ }^{25}$ Caldwell JD, Walker CH, O'Rourke ST, Faggin BM, Morris M, Mason GA. Analogies between oxytocin systems of the uterus and brain. Horm Metab Res 1996; 28: $65-74$

${ }^{26}$ Porto CS, Musto NA, Bardin CW, Gunsalus GL. Binding of an extracellular steroid-binding globulin to membranes and soluble receptors from human breast cancer cells (MCF-7 cells). Endocrinology 1992; 130: $2931-2936$

${ }^{27}$ Fortunati N, Fissore F, Fazzari A, Berta L, Benedusi-Pagliano E, Frairia R. Biological relevance of the interaction between sex steroid binding protein and its specific receptor of MCF-7 cells: effect on the estradiol-induced cell proliferation. J Steroid Biochem Mol Biol 1993; 45: $435-444$

${ }^{28}$ Hryb DJ, Khan MS, Romas NA, Rosner W. Solubilization and partial characterization of the sex hormone-binding globulin receptor from human prostate. J Biol Chem 1989; 264: 5378 -5383 
${ }^{29}$ Hryb DJ, Khan MS, Romas NA, Rosner W. The control of the interaction of sex hormone-binding globulin with its receptor by steroid hormones. J Biol Chem 1990; 265: 6048-6054

${ }^{30}$ Khan MS, Hryb DJ, Hashim GA, Romas NA, Rosner W. Delineation and synthesis of the membrane receptor-binding domain of sex hormonebinding globulin. J Biol Chem 1990; 265: $18362-18365$

${ }^{31}$ Grishkovskaya I, Avvakumov GV, Sklenar G, Dales D, Hammond GL, Muller YA. Crystal structure of human sex hormone-binding globulin: steroid transport by a laminin G-like domain. EMBO J 2000; 19: $504-$ 512

${ }^{32}$ Gershagen S, Fernlund P, Lundwall A. A cDNA coding for human sex hormone binding globulin. Homology to vitamin K-dependent protein S. FEBS Lett 1987; 220: 129-135

33 Joseph DR, Baker ME. Sex hormone-binding globulin, androgen-binding protein, and vitamin K-dependent protein $S$ are homologous to laminin A, merosin, and Drosophila crumbs protein. FASEB J 1992; 6: $2477-2481$

34 Raineri M, Catalano MG, Hammond GL, Avvakumov GV, Frairia R, Fortunati N. O-Glycosylation of human sex hormone-binding globulin is essential for inhibition of estradiol-induced MCF-7 breast cancer cell proliferation. Mol Cell Endocrinol 2002; 189: 135-143

${ }^{35}$ Fissore F, Fortunati N, Comba A, Fazzari A, Gaidano G, Berta L, Frairia R. The receptor-mediated action of sex steroid binding protein (SBP, SHBG): accumulation of cAMP in MCF-7 cells under SBP and estradiol treatment. Steroids 1994; 59: $661-667$

${ }^{36}$ Catalano MG, Comba A, Fazzari A, Benedusi-Pagliano E, Sberveglieri M, Revelli A, Massobrio M, Frairia R, Fortunati N. Sex steroid binding protein receptor (SBP-R) is related to a reduced proliferation rate in human breast cancer. Breast Cancer Res Treat 1997; 42: 227 - 234

${ }^{37}$ Marquez DC, Pietras RJ. Membrane-associated binding sites for estrogen contribute to growth regulation of human breast cancer cells. Oncogene 2001; 20: 5420-5430

38 Razandi M, Pedram A, Levin ER. Plasma membrane estrogen receptors signal to antiapoptosis in breast cancer. Mol Endocrinol 2000; 14: $1434-1447$

${ }^{39}$ Levin ER. Bidirectional signaling between the estrogen receptor and the epidermal growth factor receptor. Mol Endocrinol 2003; 17: 309 317. Epub 2002 Dec 12

${ }^{40}$ Pearson G, Robinson F, Beers Gibson T, Xu BE, Karandikar M, Berman K, Cobb MH. Mitogen-activated protein (MAP) kinase pathways: regulation and physiological functions. Endocr Rev 2001; 22: 153-183

${ }^{41}$ Santen RJ, Song RX, McPherson R, Kumar R, Adam L, Jeng MH, Yue W. The role of mitogen-activated protein (MAP) kinase in breast cancer. J Steroid Biochem Mol Biol 2002; 80: 239-256

${ }^{42}$ Freeman SM, Whartenby KA. The role of the mitogen-activated protein kinase cellular signaling pathway in tumor cell survival and apoptosis. Drug News Perspect 2004; 17: 237-242

${ }^{43}$ Kato S, Endoh H, Masuhiro Y, Kitamoto T, Uchiyama S, Sasaki H, Masushige S, Gotoh Y, Nishida E, Kawashima H, Metzger D, Chambon P.
Activation of the estrogen receptor through phosphorylation by mitogen-activated protein kinase. Science 1995; 270: 1491 - 1494

${ }^{44}$ Nakhla AM, Khan MS, Rosner W. Biologically active steroids activate receptor-bound human sex hormone-binding globulin to cause LNCaP cells to accumulate adenosine 3',5'-monophosphate. J Clin Endocrinol Metab 1990; 71: $398-404$

${ }^{45}$ Rosner W, Hryb DJ, Khan MS, Nakhla AM, Romas NA. Sex hormonebinding globulin. Binding to cell membranes and generation of a second messenger. J Androl 1992; 13: $101-106$

${ }^{46}$ Fortunati N, Fissore F, Fazzari A, Piovano F, Catalano MG, Becchis M, Berta L, Frairia R. Estradiol induction of cAMP in breast cancer cells is mediated by foetal calf serum (FCS) and sex hormone-binding globulin (SHBG). J Steroid Biochem Mol Biol 1999; 70: 73-80

${ }^{47}$ Nakhla AM, Leonard J, Hryb DJ, Rosner W. Sex hormone-binding globulin receptor signal transduction proceeds via a $G$ protein. Steroids 1999; 64: $213-216$

${ }^{48}$ Fazzari A, Catalano MG, Comba A, Becchis M, Raineri M, Frairia R, Fortunati N. The control of progesterone receptor expression in MCF-7 breast cancer cells: effects of estradiol and sex hormone-binding globulin (SHBG). Mol Cell Endocrinol 2001; 172: 31 - 36

${ }^{49}$ Fortunati N, Fissore F, Fazzari A, Becchis M, Comba A, Catalano MG, Berta L, Frairia R. Sex steroid binding protein exerts a negative control on estradiol action in MCF-7 cells (human breast cancer) through cyclic adenosine 3',5'-monophosphate and protein kinase A. Endocrinology 1996; 137: 686-692

${ }^{50}$ Catalano MG, Frairia R, Boccuzzi G, Fortunati N. Sex hormone-binding globulin antagonizes the anti-apoptotic effect of estradiol in breast cancer cells. Mol Cell Endocrinol 2005; 230: 31 - 37

${ }^{51}$ Filardo EJ, Quinn JA, Frackelton AR Jr, Bland KI. Estrogen action via the $G$ protein coupled receptor, GPR30: stimulation of adenylyl cyclase and cAMP-mediated attenuation of the epidermal growth factor receptor-to-MAPK signaling axis. Mol Endocrinol 2002; 16: 70-84

52 Berkenstam A, Glaumann H, Martin M, Gustafsson JA, Norstedt G. Hormonal regulation of estrogen receptor messenger ribonucleic acid in T47Dco and MCF-7 breast cancer cells. Mol Endocrinol 1989; 3: 22 28

53 Jensen BL, Skouv J, Lundholt BK, Lykkesfeldt AE. Differential regulation of specific genes in MCF-7 and the ICI 182 780-resistant cell line MCF7/182R-6. Br J Cancer 1999; 79: 386-392

54 Dickson RB, Thompson EW, Lippman ME. Hormones and breast cancer in vitro. Hum Cell 1989; 2: 219-230

${ }^{55}$ Frasor J, Danes JM, Komm B, Chang KCN, Lyttle R, Katzenellenbogen BS. Profiling of estrogen up- and down-regulated gene expression in human breast cancer cells: insights into gene networks and pathways underlying estrogenic control of proliferation and cell phenotype. Endocrinology 2003; 144: 4562 - 4574

56 Thiantanawat A, Long BJ, Brodie AM. Signaling pathways of apoptosis activated by aromatase inhbitors and antiestrogens. Cancer Res 2003; 63: $8037-8050$ 